\title{
SOCRATE “NATURALISTA”: GLI INDIZI DEI LOGOI SOKRATIKOI IN PLATONE E IN SENOFONTE
}

\section{Rineu Quinalia Mestrando em Filosofia pela UNIFESP}

Resumo: Este artigo centra-se em interpretar o personagem Sócrates à luz de algumas passagens de Platão e de Xenofonte e, assim, sugerir uma leitura biográfica do personagem histórico Sócrates sob a ótica do materialismo dos físicos que o precederam. Apresentaremos alguns trechos específicos do Fédon platônico e das Memoráveis de Xenofonte. A obra platônica é indiscutivelmente de fundamental importância no que se refere a indícios biográficos de Sócrates, mas não é a única. Muitas vezes menosprezada pelos estudantes, mas igualmente valiosa, é a obra de Xenofonte que, em nossa leitura, assume um papel relevante para o estudo do socratismo, representando uma imprescindível e importante luneta histórica para a difícil práxis dos estudos acerca do personagem histórico Sócrates.

Palavras-chave: Socrates. Platão. Xenofonte. História da Filosofia Antiga.

\begin{abstract}
This article focuses on intepreting the character Socrates in the light of some passages of Plato and Xenophon, and thus suggest a biographical reading of the historical character Socrates from the perspective of physical materialism that preceded it. Plato's work is undoubtedly of fundamental importance in relation to Socrates' biographical evidence, but it is not the only one. Often overlooked by students, but equally valuable, is the work of Xenophon, which, in our reading, assumes a relevant role for the study of the Socratic method, representing an essential and important historical scope for the praxis of the difficult studies concerning the historical character Socrates.
\end{abstract}

Keywords: Socrates. Plato. Xenophon. History of Ancient Philosophy. 


\section{Platone e il Fedone}

Infatti, tutti i sapienti, esaltando veramente se stessi, sono concordi nel ritenere che l'intelligenza è per noi regina del cielo e della terra.

Platone

La prima parte di questo articolo avrà lo scopo di illustrare alcuni passi dell'opera di Platone, specialmente il Fedone. Preciso innanzitutto che non ho l'intenzione di limitare l'importanza che gli scritti di Platone hanno per la ricostruzione della vita e pensiero di Socrate, ma trovo importante mettere in chiaro che questa parte del mio lavoro tratterà non soltanto del "Socrate platonico" bensì del "Platone socratico", poiché Platone nelle sue opere non ha mai un atteggiamento meramente biografico o dossografico nei confronti del figlio di Sofronisco.

È necessario sapere che nell'opera platonica troviamo un personaggio letterario e non un ritratto biografico di Socrate. Salvo rare eccezioni (il Fedone per esempio), Platone non si preoccupa di narrare la vita del suo maestro. Come Senofonte, anche egli ha voluto conservare le memorie del filosofo di Alopece, ma in un modo diverso. In questa parte, cercheremo di notare le differenze tra l'immagine di Socrate proposte dai suoi due più famosi discepoli.

Il Fedone è di fondamentale importanza per ricostruire la biografia di Socrate. Tra le opere di Platone, questa in particolare rappresenta quanto abbiamo di più prezioso per tentare di sciogliere l'affascinante enigma di Socrate, ossia la sua gioventù. Il Fedone contiene un lungo excursus nel quale Platone fa ripercorrere al suo maestro la sua autobiografia intelletuale. Qui Platone racconta i primi interessi di un giovane Socrate insoddisfatto nei confronti delle indagini naturalistiche. Platone fa dire a Socrate che non soltanto le indagini sulla natura sono ormai superate ma che quando egli stesso si imbatteva in queste ricerche "disimparava" anche quello che sapeva; la ricerca naturalistica, insomma, lo rendeva più ignorante. Dice Socrate:

Tutto quello che io sapevo con chiarezza, almeno come pareva a me e agli altri, allora da questa ricerca mi si fece oscuro a tal punto che disimparai perfino quello che prima ero convinto di sapere, sia riguardo a molte altre cose, sia riguardo alla ragione per cui l'uomo cresce $^{1}$.

\footnotetext{
${ }^{1}$ Platone, Fedone, 96c.
} 
Ciò nonostante, Platone lascia non pochi dubbi sul fatto che Socrate avrebbe conosciuto la dottrina di Anassagora, attraverso probabilmente il suo libro Sulla Natura; Prosegue Socrate: "Presi dunque i suoi libri con la più grande sollecitudine, e li lessi il prima possibile per poter, il prima possibile conoscere il meglio e il peggio" ${ }^{\text {. Anche Le }}$ Nuvole (come vedremo nell'ultima parte), testimoniano dell'interesse di Socrate per le ricerche sulla natura. Ma nel caso della commedia, Aristofane, attraverso una critica satirica, trasforma questo interesse in accuse e lo discrive come fiero e orgoglioso delle sue indagini.

È essenziale cercare di approfondire il resoconto presente nel Fedone, non solo perché i dialoghi platonici costituiscono la testimonianza "generale" più abbondante su Socrate, ma soprattutto perché il Socrate cosiddetto platonico è quello che ha esercitato la più grande influenza sul pensiero occidentale. Si presenta qui un'analisi sintetica del Fedone.

Provare a ricostruire brevemente il percorso degli ultimi giorni della vita di Socrate attraverso alcuni dialoghi di Platone è impresa importante e utile: dopo l'Eutifrone, che ha luogo nel portico del tribunale dove Socrate ha letto per la prima volta la notizia della sua condanna, si ha l'Apologia, nella quale egli viene condannato in tribunale; successivamente abbiamo il Critone, dove Socrate è già in carcere e incontra il suo discepolo e amico Critone che tenta invano di convincerlo a evadere. Ed infine arriviamo al Fedone, dove Socrate in carcere ha il suo ultimo colloquio con gli amici, questa volta nel giorno della sua morte $^{3}$, nella primavera del 399 a.C.

Secondo G. Giannantoni ${ }^{4}$, il Simposio e il Fedone inaugurano la fase più matura della produzione filosofica di Platone. Qui infatti possiamo trovare oltre alla sezione che attesta l'iniziale vicinanza di Socrate ad Anassagora e al suo pensiero, anche celebri passi sul concetto di amore ${ }^{5}(60 \mathrm{~b})$ e dimostrazioni dell'immortalità dell'anima $(69 \mathrm{e}-$

\footnotetext{
${ }^{2}$ Ivi, $98 \mathrm{c}$.

${ }^{3}$ Secondo Senofonte, Cfr. Memorabili, IV, 8, Socrate "fu costretto a vivere in carcere trenta giorni dopo la sentenza" perché non potevano avere luogo esecuzioni capitali fino al ritorno della nave inviata a Creta come ex voto nel rispetto dell'antica tradizione. Fedone, 58b.

${ }^{4}$ Cfr. G. Giannantoni, Dialogo Socratico e nascita della dialética nella filosofia di Platone (v. bibliografia).

${ }^{5}$ Nel Fedone, Platone presenta una definizione dell'amore simile a quella del racconto sull'originaria natura degli uomini di Aristofane nel Simposio (189e). Mentre nel Simposio Platone mette in bocca ad Aristofane il mito degli esseri androgini, nel Fedone appena Santippe se ne va dal carcere, Socrate dice ai suoi amici che gli amanti vogliono stare sempre insieme e se qualcuno insegue e prende uno dei due, è pressoché costretto a prendere sempre anche l'altro.
} 
77d); inoltre, viene pienamente esplicitato il fondamento della sua dottrina della reminiscenza, ossia, una delle colonne del pensiero platonico, la teoria delle Idee (73c 74e). La filosofia è definita come "esercizio di morte", definizione che risuona per quasi tutta la storia della filosofia occidentale ${ }^{7}$ da allora fino ai nostri giorni.

Platone, nelle sue opere, non si riferisce mai a sé stesso, tranne in due di esse, l'Apologia di Socrate ed il Fedone. Quando il personaggio Fedone dice che "Platone, credo era malato", egli segna uno dei più intriganti passaggi di tutta l'opera di Platone. Nel Fedone, più che in tutti gli altri dialoghi, si rivela la praxis dialectica di Platone, che consiste nel creare e nascondersi dietro al suo personaggio; a Socrate sono così fatte esprimere idee filosofiche strettamente platoniche.

Nel Fedone Platone fa enunciare al proprio maestro il cuore del suo pensiero, ossia le sue convinzioni metafisiche. Secondo un aneddoto trasmesso dall'antichità, una volta Socrate, mentre ascoltava Platone leggere il Liside ${ }^{10}$, avrebbe detto: "Per Eracle, quante bugie questo ragazzo mi fa dire" ${ }^{11}$.

È importante tenere sempre presente che il Socrate del Fedone, e di tutta l'opera di Platone, diventa sempre, oltre che personaggio dialogico, la sua fondamentale ed emblematica dramatis persona.

\subsection{Socrate, il discepolo di Anassagora}

Di tutta l'opera platonica il Fedone rappresenta senza alcun dubbio il principale documento per edificare una teoria sulle radici naturalistiche di Socrate. Due sono i motivi principali. Il primo è chiaramente la ricchezza del contenuto della critica

\footnotetext{
${ }^{6} \mathrm{Cfr}$. Fedone, 61d, 62b e-d, 66e.

${ }^{7}$ Questa definizione ha avuto notevoli influssi in Cicerone, che nelle sue Tusculanae, I 30, scriveva: "tota enim philosophorum vita $[\ldots]$ commentatio mortis est"; in Montaigne abbiamo tutto un capitolo nel primo libro dei suoi saggi intitolato "Filosofare è imparare a morire". Infine, mi pare pertinente richiamare anche il tema heideggeriano dell'"essere per la morte".

${ }^{8}$ Cfr. Platone, Apologia di Socrate 34a.

${ }^{9}$ Cfr. Fedone, 59b. Questo passo potrebbe rinviare a due ipotesi: la prima, secondo la quale Platone sarebbe stato effettivamente affetto da un malore fisico; la seconda, secondo la quale questo "malore" sarebbe una metafora per esprimere disgusto, tristezza e delusione, in conseguenza del fatto che il suo maestro era stato condanato ingiustamente a morire.

${ }^{10}$ Nel Liside Platone tratta del tema dell'amicizia. Il Liside non è un dialogo di facile e sicura collocazione cronologica, infatti gli studiosi lo hanno analizzato basandosi su due distinte teorie; la prima suggerisce che essendo esso simile al Carmide dal punto di vista stilistico-letterario e della problematica filosofica, l'opera farebbe parte degli scritti giovanili; la seconda invece sostiene che il Liside va collocato alla soglia dei dialoghi di maturità, essendo stato composto subito dopo il Simposio ed il Fedro, i quali rispondono alle aporie dei dialoghi della gioventù.

${ }^{11}$ Diogene Laerzio, Vite dei filosofi, III, 35.
} 
platonica alla teoria di Anassagora (96a - 99d); ne deriva un parallelo ricco di significato tra il pensiero di Socrate e quello del filosofo di Clazomene.

Il secondo motivo è costituito dal fatto che il Fedone è un'opera pienamente matura $^{12}$. Platone vi raggiunge il punto massimo della sua indipendenza filosofica e le indicazioni che egli fornisce possono essere considerate affidabili proprio alla luce del punto di vista che egli ha conseguito.

\subsection{1. "Stammi pure a sentire che te la narro"13}

Con queste parole Platone inizia la sua esposizione critica sulla inadeguatezza dell'indagine dei filosofi naturalisti. Nel Fedone il discorso sulla fase naturalistica di Socrate ha inizio a partire da 96a:

Io da giovani nutrii un desiderio vivissimo di possedere quella scienza che chiamano 'indagine sulla natura'. Infatti mi sembrava una cosa straordinaria sapere quali sono le cause di ciascuna cosa, ossia sapere perché ciascuna cosa si genera, perché si corrompe e perché esiste. $\mathrm{E}$ molte volte mi rivolsi da un capo all'altro nelle mie indagini, esaminando, in primo luogo, problemi di questo genere: quando il caldo e il freddo entrando in decomposizione, si formano gli essere viventi. Poi, esaminai i processi di corruzione delle cose e i fenomeni del cielo e della terra.

Ma è più avanti $(97 \mathrm{c})$ che la testimonianza prende forma definitiva, nel momento in cui il Socrate messo in scena da Platone racconte che un giotno avrebbe udito un tale leggere un libro che affermava essere di Anassagora. Intorno all'identità di questo questo "tale", si sono sviluppate alcune teorie: questa figura potrebbe essere infatti identificata con Archelao o con lo stesso Anassagora. Platone nel suo dialogo non ne chiarisce l'identità, ma è Diogene Laerzio (II, 16,17 ) a riferire che si tratterebbe di Archelao $^{14}$. In effetti, non ci sono notizie di un incontro tra Anassagora e Socrate, ed è plausibile che la notizia trasmessa da Diogene sia fededegna.

Il periodo di formazione intelletuale di Socrate è abbastanza oscuro dal punto di vista della ricerca storica. Sono arrivate fino a noi soltanto poche righe ed alcuni

\footnotetext{
${ }^{12} \mathrm{Si}$ vede la nota 21.

${ }^{13}$ Platone, Fedone, 96a.

${ }^{14}$ Archelao di Atene o di Mileto è stato discepolo di Anassagora. Secondo la tradizione Socrate sarebbe stato il suo principale allievo nonchè il suo "amasio". Per ulteriori notizie su Archelao, vedere Diogene Laerzio, Vite dei filosofi, II,16-17 e Diels, Die Fragmente der Vorsokratiker, 60.
} 
aneddoti ma se ci fidiamo di una tardiva testimonianza di Porfirio ${ }^{15}$, il discepolo di Anassagora Archelao, avrebbe conosciuto un Socrate diciassettenne ad Atene (nel 452 a.C.). Da quel momento sarebbe nato il "filosofo" Socrate, poichè il suo maestro lo avrebbe avviato non solo alle indagini naturalistiche, ma anche alle discussioni morali, visto che secondo Archelao al giovane Socrate mancava l'equilibrio e la moderazione.

Dal momento che sappiamo, non solo attraverso la possibile testimonianza di Platone ma attraverso altre fonti ${ }^{16}$, che Socrate avrebbe avuto a che fare in gioventù con il pensiero naturalistico, possiamo capire che tutta la testimonianza del Fedone platonico ha un fondamento storico.

\subsubsection{Conclusione}

In questa breve esposizione del Fedone ho voluto suggerire che nonostante il Socrate platonico non debba essere preso come modello di fedeltà storica, la testimonianza che Platone fornisce di un giovane Socrate interessato alle ricerche sulla natura è abbastanza ricca ed importante. Tenendo presente la maturtà di Platone nel momento di composizione del Fedone (dopo il 387 a.C.) questa testimonianza ha un notevole peso storico.

Ma Platone, nel passo sopra studiato del Fedone (96a-97c), fornisce comunque un resoconto critico delle tendenze con cui si confrontò il giovane Socrate, testimoniando del fatto che egli restò deluso dalle sue prime indagini (cfr. 96c).

Platone, nella sua critica alla inadeguatezza delle ricerche sulla natura, vuole raggiungere due scopi: in primis, la sua intenzione è discolpare Socrate e fugare qualsiasi dubbio su una sua supposta mancanza di rispetto verso gli dèi patri e sul fatto che egli abbia introdotto nuove credenze fisiche: infatti nell'Eutifrone (3b) Platone dice che gli accusatori l'ho avrebbero accusato di essere un creatore di nuovi dèi, e che in quanto tale non credeva più ai vecchi. Successivamente, Platone attraverso questa sua critica vuole attaccare un tradizionale metodo di ricerca filosofica, per introdurne un altro di stampo metafísico: un metodo questo che, come testimonia Aristotele (nella sua Metafisica), va esclusivamente attribuito a Platone, non potendo essere attribuito, storicamente, a Socrate.

\footnotetext{
${ }^{15}$ Cfr. 60 A 13 D.-K.

${ }^{16}$ Diogene Laerzio, Vite dei filosofi e Diels-Kranz, Die Fragmente der Vorsokratiker.
} 
Detto questo, è forse possibile suggerire un dilemma ermeneutico. Ci si può chiedere se Platone, nel Fedone, cerchi di proporre attraverso Socrate, una teoria dell'immortalità dell'anima di stampo orfico-pitagorico, oppure una teoria di stampo anassagoreo. In un importante frammento di Sulla Natura, dove Anassagora affermava che: "nessuna cosa nasce o muore, ma appartire dalle cose che sono si ha comporsi e il dividersi. E così dovrebbero corretamente chiamare il nascere comporsi e il morire dividersi". ${ }^{17}$

Questa sintesi del Fedone, insieme alla sintesi della prossima "voce socratica", quella di Senofonte, ha l'obiettivo di fornire gli elementi propedeutici ad una più solida analisi delle Nuvole.

\section{Senofonte e i Memorabili}

Il Socrate platonico è propriamente una caricatura, invece i Memorabili di Senofonte danno un'immagine di Socrate realmente fedele, che è esattamente intelligente, bisogna però saper leggere questo libro.

(Friedrich Nietzsche, Umano troppo umano, I e Frammenti postumi, 1876-1878)

Edificare ipotesi o teorie sul Socrate storico e le sue radici naturalistiche, come già si è detto, non è affatto un compito facile. Come, e dove dobbiamo guardare per estrarre le informazioni più affidabili? Oltre alle Nuvole, le due principali fonti storiche sono: Platone ${ }^{18}$, con la sua testimonianza nel Fedone, e Senofonte, con le sue testimonianze nei Memorabili. La complessità di questo nodo storico è colossale, Schleiermacher nel suo Über den Wert des Sokrates als Philosophen (1818), di fronte a questo problema, suggeriva di non fidarci esclusivamente delle testimonianze di Senofonte o di Platone, bensì di muoverci diplomaticamente tra di loro.

Le pagine di Senofonte rappresentano una perpetua testimonianza dei sentimenti di riverenza e di ammirazione che Socrate era capace di suscitare nell'animo di quanti lo avvicinassero. L'intenzione dei Memorabili è quella di difenderne l'immagine, anche se in maniera postuma, di cittadino estremamente patriottico, pio, giusto e onesto, che compiva i sacrifici agli dèi della città, un leale amico, obbediente verso tutti i suoi doveri di cittadino, ed anche un grande e coraggioso soldato, protagonista delle battaglie

\footnotetext{
${ }^{17}$ Anassagora, Fr. 4.

${ }^{18}$ Platone viene nominato soltanto una volta in tutta l'opera senofontea. Questo accade nei Memorabili III, 6,1 .
} 
in Tracia a Potidea (433 a.C) contro i Tebani, in Beozia a Delio (424 a.C), e poi di nuovo in Tracia, questa volta preso a Anfipoli (422 a.C), dove dimostrò grandiosa temperanza, forza, valore ${ }^{19}$ e un straordinario coraggio.

La praxis dialectica di Senofonte nei suoi scritti socratici ${ }^{20}$ si discosta da quella di Platone: mentre il filosofo esprime molto spesso i suoi pensieri attraverso la voce di Socrate, Senofonte lo fa meno. Ad esempio, nel passo che segue, tratto del primo libro dei Memorabili, Senofonte si esprime in prima persona per raccontare il quotidiano del suo maestro. Questa tecnica si delinea permanente in tutta l'opera:

Inoltre viveva sempre sotto gli occhi di tutti. Al mattino infatti si recava nei portici e nei ginnasi quando l'agorà era piena di gente, si poteva vederlo là, e per tutto il resto della giornata si trovava dove avrebbe incontrato più gente possibile. Per la maggior parte del tempo parlava $^{21}$.

Senofonte, nei Memorabili, sviluppa una vera e propria difesa appassionata di Socrate, affermando che non è vero che egli non credesse negli dèi della città. Infatti all'inizio dei Memorabili è sottolineata la devozione di Socrate e si afferma che sia in privato che in pubblico il filosofo compiva sempre i dovuti sacrifici agli dèi ${ }^{22}$. Così facendo, Senofonte voleva sostenere che l'accusa di avere introdotto nuove divinità, ad esempio quella che troveremo nelle Nuvole di Aristofane, non era altro che una calunnia. Senofonte continua affermando che era parimenti falso che Socrate corrompesse i giovani ${ }^{23}$. Senofonte difende Socrate sostenendo che i giovani (Crizia e Alcibiade erano appunto stati discepoli di Socrate) non erano diventati i peggiori cittadini della polis per colpa degli insegnamenti di Socrate, bensì a causa di un preesistente carattere cattivo e ambizioso, cercando cosi di sollevare Socrate dalla responsabilità dei loro atteggiamenti ${ }^{24}$.

\footnotetext{
${ }^{19}$ Ed infatti per esempio, Diogene Laerzio contrapone I Memorabili ad alcuni dialoghi di Platone: il Lachete (sul coraggio), al Critone (sul dovere) e il Carmide (sulla temperanza).

${ }^{20}$ I Memorabili, l'Economico, L'Apologia e il Simposio. Cfr. supra, nota 7.

${ }^{21}$ Cfr. Senofonte, Memorabili, I, 10.

${ }^{22} \mathrm{Cfr}$. Ivi, I, 2.

${ }^{23}$ Secondo Senofonte (Cfr. Memorabili., I,2,12) l'accusa si fondava sul fatto che tra i discepoli di Socrate vi fossero personaggi Crizia e Alcibiade. Il primo fu il più avido e violento di tutti coloro che si erano impegnati nella tirannica oligarchia ateniese, mentre il secondo fu il peggiore, il più violento e arrogante tra i sostenitori del fronte democratico di Atene.

${ }^{24}$ Cfr. Senofonte, Memorabili I,2.
} 
Il Socrate senofonteo è un Socrate pratico, estremamente preoccupato delle semplici faccende morali del quotidiano. I Memorabili sono un vero manuale di vita pratica. Socrate attraverso la penna di Senofonte si trasforma in un maestro di vita che girando per le vie, case, mercati, piazze e botteghe della polis emana saggezza. A confermare questa immagine possono bastare due esempi.

Nel primo Socrate promuove l'armonia di due fratelli, Cherefonte e Cherecrate che vivevano in disaccordo:

Dimmi o Cherecrate, non appartieni anche tu, vero, a quella specie di uomini che considerano il denaro più vantaggioso dei fratelli? Anche se il denaro è senza intelligenza, mentre il fratello è dotato di ragione, quello ha bisogno di aiuto, questo è in grado di darne e inoltre di denaro ce n'è molto, di fratelli uno solo ${ }^{25}$.

Nel secondo, da attento padre, cura l'ingratitudine e l'insofferenza del suo primogenito $^{26}$, Lamprocle, quando egli gli confessa di non sopportare più la durezza di sua madre Santippe:

Quanti affanni pensi di averle procurato da piccino facendo le bizze, gridando e facendo dispetti, di notte e di giorno? Quante preoccupazioni le hai dato quando ti ammalavi? Devi sapere che le cose che tua madre ti dice, le dice non solo senza l'intenzione di farti male, ma anzi perchè vuole che tu abbia tanti beni ${ }^{27}$.

Platone dipinge un ritratto filosofico fine e molto complesso di Socrate; invece Senofonte coglie e apprezza anch'egli il senso della riflessione filosofica di Socrate, ma sottolinea soprattutto il valore del suo insegnamento per la prassi quotidiana, rendendo così i Memorabili un documento di estremo valore storico.

\subsection{Memorabili: e l'influenza di Anassagora nel discorso socratico}

Come si è detto, l'intenzione delle opere cosidette socratiche di Senofonte è quella di cancellare l'immagine di un Socrate sovversivo rispetto alle leggi e ai costumi

\footnotetext{
${ }^{25} \mathrm{Ivi}, \mathrm{II}, 3$.

${ }^{26}$ Socrate aveva tre figli: Lamprocle, Sofronisco e Menesseno. Secondo una tradizione risalente al IV secolo, Sofronisco e Menesseno non erano figli di Santippe, ma di una sua precedente moglie, Mirtò.

${ }^{27}$ Senofonte, Memorabili, II,2.
} 
della polis. Ed infatti Senofonte, già all'inizio dei Memorabili, dice chiaramente che Socrate, a differenza di tutti gli altri pensatori, non si è mai interessato alle indagini sulla natura né ai fenomeni celesti; addirittura, egli si spinge a dire che Socrate riteneva coloro che si sono occupati di tutto ciò nient'altro che dei folli ${ }^{28}$. Vale la pena riportare per intero questo passaggio chiave:

Socrate, per la maggior parte del tempo, parlava e, a chi lo desiderava, era possibile ascoltarlo. Eppure nessuno mai lo vide o sentì fare o dire niente di irreligioso o empio. E infatti non trattava della natura di tutte le cose alla maniera della maggior parte degli altri pensatori, indagando com'è fatto quello che i sapienti chiamavano kosmos (universo ordinato) e per quali leggi necessarie avvenga ciascuno dei fenomeni celesti, ma indicava come matti quelli che credono di poter conoscere e capire, i fenomeni celesti ${ }^{29}$.

Questo passagio ci porta al cuore della dimensione non soltanto del quotidiano di Socrate, ma anche degli interesse del Socrate senofonteo. Senofonte prosegue affermando che Socrate si chiedeva prima di tutto se questi pensatori facessero la cosa giusta impegnando la loro mente in tali argomenti fisici. Il Socrate di Senofonte credeva che i sapienti non dovrebbero sprecare il loro tempo perdendosi in riflessioni sull'origine, il funzionamento o l'importanza dei corpi celesti o ricercando di svelare i misteri del kosmos. Questi pensatori, secondo Socrate, sbagliavano a trascurare le cose umane a favore di quelle divine. "Forse pensavano di sapere già abbastanza" 30 affermava Socrate forse con ironia.

Ma le cose non sono cosí semplici come potrebbero apparire. Nei libri dei Memorabili si trovano, infatti, due importanti indizi che provano un interesse di Socrate per l'analisi della natura, attraverso l'osservazione dei corpi celesti.

Su questo avvicinamento di Socrate agli interessi dei naturalisti abbiamo un contributo di David Sedley. Nella sua opera Creationism and its critics in antiquity, (2007), Sedley dedica un interessante capitolo al Socrate di Senofonte, nel quale cerca di avvicinare Socrate ad un altro fisico ${ }^{31}$, Diogene di Apollonia ${ }^{32}$.

\footnotetext{
${ }^{28}$ Cfr. Ivi, I, 11.

${ }^{29}$ Ivi, I, 10,11,12

${ }^{30} \mathrm{Ivi}, \mathrm{I}, 12$.

${ }^{31}$ D. Sedley, Creationism and its critics in antiquity, III, 1 (cfr. Bibliografia).

32 È stato un filosofo naturalista molto famoso, secondo Antistene è stato alunno di Anassimene. Contemporaneo di Anassagora, il punto fondamentale della sua dottrina aveva l'aria come elemento originario.
} 
L'intepretazione del Socrate dei Memorabili è assai complessa, viste le contraddizioni presenti nel medesimo testo. Mentre all'inizio come abbiamo visto (cfr. I, 11) Senofonte afferma che Socrate non si era mai preoccupato di condurre indagini sul kosmos e sull'importanza dei corpi celesti, alcuni libri dopo lo stesso Senofonte ci mostra il nostro filosofo nell'atto di consigliare a un discepolo l'importanza di essere un esperto in astronomia e di studiare le stelle e i corpi celesti (cfr. IV, 7,5). Socrate prosegue affermando di non essere del tutto ignaro della conoscenza dei cieli. Possiamo vedere chiaramente questa caratteristica in un dialogo fra Socrate ed Eutidemo, nel quale il filosofo appare abbastanza informato proprio sui corpi celesti, sul sole e sulla luna, e sul loro funzionamento e la loro importanza:

Dimmi, o Eutidemo ma tu lo sai quanto abbiamo bisogno della luce che gli dei ci danno? Ché, se non l'avessimo saremmo come ciechi. Inoltre abbiamo bisogno anche di una pausa, e per questo esiste la notte, tempo bellissimo per il riposo. E siccome il sole con la sua luce rischiara per noi i momenti della giornata ${ }^{33}$, la notte, per come è scura, rende difficile distinguere le cose per ciò abbiamo accese le stelle. Inoltre la luna ci rende riconoscibili non solo le parti della notte, ma anche del mese ${ }^{34}$.

Interessante è anche l'analisi di Socrate sui movimenti del sole tra le stagioni dell'anno. Socrate ci spiega che dopo l'inverno il sole ritorna, e viene la primavera per fare maturare alcuni frutti della stagione, ma comunque il calore dal sole rimane fiacco, poiché l'astro non si avvicina molto alla terra come nell'estate. Esso si mantiene distante perché, se si avvicinasse troppo, potrebbbe danneggiarci riscaldandoci più del necessario, ma non si allontana troppo perché, se facesse così, moriremmo di freddo. Il sole si avvicina gradualmente e poi gradualmente si allontana, perchè non possiamo resistere né al caldo né al freddo (cfr. IV,3,8). Alla fine di questa lezione, riflette il suo discepolo Eutidemo: "Sì, per Zeus! Anche questi fenomeni sembrano verificarsi perfettamente nell'interesse degli uomini" ${ }^{35}$. Anassagora aveva affrontato il tema delle fasi degli astri. Vediamo ad esempio il frammento 13 di Sulla Natura.

[...] E quali stavano per essere e quali erano che ora non sono e quante ora sono e quali saranno tutte ordinò l'Intelletto e la rotazione che

\footnotetext{
${ }^{33}$ Il giorno per gli ateniese era diviso in 12 parti che si calcolavano in base all'ombra proietata dall'asta dello orologio solare, istallato ad Atene.

${ }^{34}$ Senofonte, Memorabili, IV,3.

${ }^{35} \mathrm{Ivi}, \mathrm{IV}, 3,9$.
} 
compiono le stelle e il sole e la luna e l'aria e l'etere. Proprio la rotazione determinò il separarsi e si separa dal rado il denso e dal freddo il caldo e dall'oscuro il luminoso e dall'umido all'asciutto.

Un altro interessante indizio compare nei Memorabili. Lo troviamo nello stesso primo libro, dove possiamo vedere un Socrate nel mezzo di una discussione con Aristodemo, nella quale egli accenna ad un termine cruciale del pensiero di Anassagora, ossia ad un supposto "intelletto" presente nell'universo.

Caro Aristodemo, renditi conto che anche la tua mente, stando in te, dirige il tuo corpo come vuole. Bisogna pensare allora che l'intelletto che c'è nell'universo disponga ogni cosa secondo il suo piacere, l'intelletto divino è capace di prestare attenzione a tutte le cose insieme ${ }^{36}$.

Ancora una volta, è possibile avvicinare senza molta difficoltà quest'ultima idea di Socrate al frammento 13 di Anassagora: "quante cose hanno vita, quelle più grandi e quelle più piccole, tutte domina l'intelletto [...] le cose composte e quelle separate e quelle divise tutte l'intelletto conobbe" ${ }^{, 37}$.

Questi brevi esempi senza alcun dubbio non sono sufficienti per poter affermare che Senofonte attesti con certezza un'influenza di Anassagora sul pensiero di Socrate. Si tratta, però, di paralleli interessanti che vanno tenuti in considerazione in vista della valutazione complessiva della questione.

\section{Conclusione}

In questo articolo ho cercato di illustrare le difficoltà che i Memorabili pongono a chi voglia ricostruire un'immagine omogenea di Socrate. Ho anche cercato di evidenziare un differente punto di vista.

In primis, il Socrate che emerge dalle pagine di Senofonte non è un personaggio dedito esclusivamente alla speculazione filosofica come quello di cui parla Platone. È piuttosto un uomo semplice, un cittadino esemplare che rispetta le leggi e venera gli dei, un maestro di vita pratica, di amor patrio e di onestà morale, che suggerisce le virtù stimolando in chi lo ascolta la riflessione sul proprio agire. I Memorabili restano tuttora

\footnotetext{
${ }^{36} \mathrm{Ivi}, \mathrm{I}, 4,17$

${ }^{37}$ Anassagora, Fr.13.
} 
una grandiosa sorgente di informazione sul personaggio Socrate. Pur tuttavia l'analisi di questa opera non è affatto semplice. Emergono alcune incoerenze, non ultima, la supposta influenza di Anassagora sul pensiero di Socrate, che ho voluto dimostrare nell'ultima parte.

Ma, con tutto ciò, rimane sempre il dubbio sull'importanza della testimonianza di Senofonte: è possibile che la sua stessa modesta intelligenza filosofica sia, paradossalmente, garanzia dell'obiettività del suo resoconto.

\section{FONTI:}

ARISTOFANE, Commedie 1, a cura di G. Mastromarco, UTET, Torino 2007.

DIOGENE LAERZIO, Vite dei filosofi, a cura di M. Gigante, Laterza, Bari 1998.

PLATONE, Tutti gli Scritti, a cura di G. Reale, Bompiani, Milano 2000.

SENOFONTE, Economico, BUR, Milano 2003

SENOFONTE, Memorabili, a cura di A. Santoni, BUR, Milano 1989.

\section{BIBLIOGRAFIA DI RIFERIMENTO}

ADORNO F., Introduzione a Socrate, Laterza, Bari 1978.

BENOIT H., Sócrates o nascimento da razão negativa, Ed. Moderna, São Paulo 1996.

DORION L.A., Compreender Sócrates, Ed. Vozes, Rio de Janeiro 2006.

FERRARI F., Socrate tra personaggio e mito, BUR, Milano 2007.

GIANNANTONI G., Socrate, Tutte le testimonianze: da Aristofane e Senofonte ai padri cristiani, Laterza, Bari 1971

JAEGER W., Paideia. La formazione dell'uomo Greco, Bompiani, Milano 2003.

MAGALHAES-VILHENA V., Le problème de Socrate: le Socrate historique et le Socrate de Platon, Presses Universitaires de France, Paris 1952.

MOSSÉ C., O processo de Sócrates, Ed. Jorge Zahar, Rio de Janeiro 1990.

OTTO W.F., Socrate e l'uomo greco, a cura di A. Stavru., Marinotti Edizioni, Milano 2005 . 